\title{
Extracting Causal Claims from Information Systems Papers with Natural Language Processing for Theory Ontology Learning
}

\author{
Roland M. Mueller \\ Berlin School of Economics and Law \\ roland.mueller@,hwr-berlin.de
}

\author{
Sebastian Huettemann \\ Berlin School of Economics and Law \\ sebastian.huettemann@gmail.com
}

\begin{abstract}
The number of scientific papers published each year is growing exponentially. How can computational tools support scientists to better understand and process this data? This paper presents a softwareprototype that automatically extracts causes, effects, signs, moderators, mediators, conditions, and interaction signs from propositions and hypotheses of full-text scientific papers. This prototype uses natural language processing methods and a set of linguistic rules for causal information extraction. The prototype is evaluated on a manually annotated corpus of 270 Information Systems papers containing 723 hypotheses and propositions from the AIS basket of eight. F1results for the detection and extraction of different causal variables range between 0.71 and 0.90. The presented automatic causal theory extraction allows for the analysis of scientific papers based on a theory ontology and therefore contributes to the creation and comparison of inter-nomological networks.

Keywords: Causal Relationship Extraction, Theory Ontology Learning, Natural Language Processing, Theory, Causality
\end{abstract}

\section{Introduction}

There is an exponential increase in the number of scientific papers published each year [37] containing countless theories and causal claims. The behavior of humans, teams, and organizations that are affected by Information Systems is therefore described by hundreds of different theories in various disciplines $[12,31]$. These theories often have minor or major variations and are evaluated and tested in different settings. Understanding and evaluating these internomological networks [33] is becoming more timeconsuming and difficult-especially if adjacent disciplines shall be included in the analysis [29].

Causal propositions and hypotheses are an important part of many Information Systems theories $[17,18,38]$. Combining, interlinking and building upon these theories is a major aspect of scientific progress. However, a researcher who wants to find, for example, all the influencing factors for the success of an e-learning initiative or the potential causes for knowledge sharing in a knowledge management system must still rely on a manual search in order to find connections, because he or she is only supported by existing literature databases with full-text search and co-citation analysis.

An automated procedure that detects and extracts hypotheses and propositions as the main theoretical content from scientific publications would support the combination and interlinking of theories. Therefore, at least two things are necessary: (1) a structured description of theoretical claims in the form of a theory ontology or meta-model $[38,39]$, which helps isolating the elements (e.g. cause and effect) of a theory and making it possible to connect them with complementary research from other publications and (2) a software-prototype that is able to automatically detect and extract the relevant contents from scientific publications into the given structure.

In this paper, a software-prototype is introduced that integrates with the meta-model of Mueller [38, 39] and is able to detect and extract hypotheses and propositions from scientific papers. Because theoretical propositions in Information Systems and other fields like management or social sciences often have not just causes and effects, but also moderators, mediators, conditions, and signs (causal directions), this prototype is developed for and tested on extracting causal theories out of papers belonging to these domains. To the best of the authors' knowledge, this combination of causal extraction tasks has not yet been addressed.

The remaining part of the paper is structured as follows: Section 2 describes the problem of causal extraction, defines theoretical causal claims, and discusses ontologies for causal theories. Section 3 presents related work regarding rule-based and machine-learning based approaches for causal relationship extraction. Section 4 describes CauseMiner, a software-prototype that applies a rulebased natural language processing approach. Section 5 
contains the evaluation of this prototype followed by the conclusion and ideas for future work.

\section{Problem Description}

Gregor [17:620] distinguishes between different means of theory representation: (a) words, (b) mathematical terms, (c) symbolic logic, (d) diagrams, (e) tables, or (f) graphically. This paper focuses on the extraction from natural language (a: words). This extracted information should then be translated and represented based on a theory ontology or theory metamodel [38]. Gregor [17] did not mention theory ontologies or formal meta-models for theories. In her classification of theory representations, it is closest to (c) symbolic logic. From a formal theory representation, diagrams (d) can be generated [38]. However, not just visualization but also causal reasoning is supported by a theory ontology [38, 39]. We define the main causal theoretical claims of a paper as all sentences that (a) are part of the theory presented in the paper and (b) have at least implicitly a causal meaning (which might be causally ambiguous).

Not all causal sentences are main causal claims and therefore relevant for further analysis. Excluded are causal sentences that state other causal claims that are unrelated to the theory, for example, that a specific circumstance of the analyzed organization affected the choice of research methods or that the relevance of the research question caused the author to analyze the research problem. In Information Systems papers, main causal claims are typically described as hypotheses or propositions. Therefore, the proposed software prototype only analyzes and extracts hypotheses and propositions.

Some hypotheses, as shown in Figure 1, do not explicitly state a causal relationship between constructs but just a relationship, even if the constructs are (causally) linked in the structure equation model (SEM). According to Pearl [41] an SEM should be interpreted causally. Therefore, we also include causally ambiguous hypotheses and rather err on including too many than too few hypotheses in our analyzes.

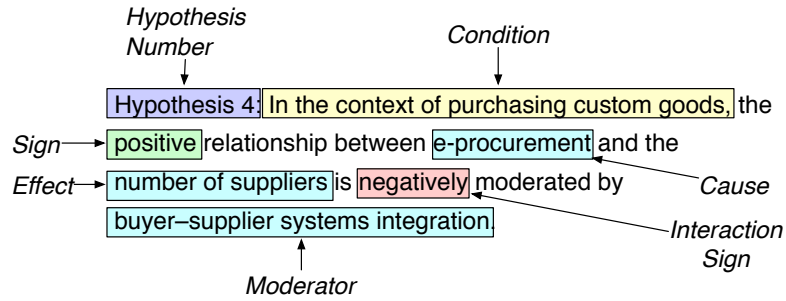

Figure 1. Example of a Hypothesis [13]
The goal of CauseMiner is the extraction of a causal theory's structure from a paper. This structure is based on the meta-model of causal theories of Mueller $[38,39]$. Figure 1 shows an example of a hypothesis from an Information Systems paper with different parts that should be extracted [13].

The extraction of theoretical structures from natural language is difficult because of (a) the detection of hypothesis and proposition sentences within the paper, (b) the numerous different possible expressions in natural language of the same logical causal structure, (c) an unclear description of the theory, (d) an incomplete description caused by e.g. omitting the scope of theory or using acronyms for variable names (e) the use of non-causal language for causal relationships, and (f) the use of different names for the same construct or the same name for different constructs [30]. Also, (g) scientific papers are often imprecise and their description of theories is sometimes incomplete and ambiguous [49]. Therefore, causal extraction with text-mining from natural language is a challenging task.

For each hypothesis in a scientific paper, CauseMiner tries to extract the following parts: hypothesis number, hypothesis, cause, effect, sign (causal direction), moderator, mediator, interaction sign, and condition. These parts are consistent with the description of theory parts by Gregor [17], Weber [49], and Mueller [38] and supported by an analysis of example hypotheses in Information Systems papers.

Cause and effect are constructs that are linked by a causal proposition or hypothesis. A typical construct in Information Systems research is e.g. "perceived ease of use'. The sign describes the relation between cause and effect and can have the following values: +, -, ?, 0 [38]. A positive sign $(+)$ means increasing the value of the cause makes a higher value of the effect more likely even if all other constructs are hold constant. A negative sign (-) is to interpret vice versa. Question marks (?) indicate an ambiguous influence whereas zeroes (0) stand for no direct causal effect at all [38]. Some hypotheses explicitly mention that there is no direct causal effect (sign 0) between two constructs. This is implicitly assumed between all constructs in a theory that are not directly linked with a hypothesis [38]. A moderator is a variable that affects the direction and/or strength of the relation between an independent and a dependent variable [38]. The mediator is a construct that explains the relation between an independent and a dependent variable. Moderator variables indicate when specific effects will hold [8], i.e. an additional variable that influences the strength of the relation between cause and effect. Mediator variables explain how or why these effects take place [8], i.e. the causal chain between cause and 
effect goes through the mediator-variable. Conditions refer to a theory's scope or boundary. These statements of boundaries show the limits of generalization [17], e.g. only under a certain condition, there is a relationship between two variables.

\section{Related Work}

Research in causal relationship extraction can broadly be divided into two categories: rule-based methods and machine learning methods [6]. Rulebased methods refer to the application of pre-defined rules to natural language text. These rules can consist of encoded grammatical structures that incorporate causal keywords, e.g. $<N P 1$ verb NP2> [15]. Thereby, they enable the detection of explicitly or implicitly stated causal relationships. Machine learning methods refer to the application of computer-based statistical learning techniques. One example for this is to train a classifier with manually annotated text examples of causal relationships. Based on this training, a classifier might be able to identify underlying rules by applying different statistical methods [6].

Li \& Larsen [33] used a rule-based system for detecting sentences as hypotheses and machine learning for construct (i.e. variable) extraction in Information Systems papers. For the rule-based hypotheses detection task, they achieved 85.39\% precision, $84.64 \%$ recall, and $84.99 \%$ f-measure. For the machine learning approach of variable extraction, they achieved $74.65 \%$ precision, $69.59 \%$ recall, $72.02 \%$ f-measure, and $89.92 \%$ accuracy. They used the extracted constructs for theory integration. Because the constructs are not labeled as cause and effect, their approach is not directly considered as causal relationship extraction.

The first publications on causal information extraction are from Khoo et. al [24, 26, 27], who worked on detecting explicitly expressed causal relationships in natural language text. They developed an automatic method based on linguistic cues for identifying and extracting cause-effect information from Wall Street Journal text. This method provided an extensive collection of linguistic patterns designed to identify causal relationships within a sentence and also in adjacent sentences [24]. Khoo et. al [27] focused on the domain-independent aspect of causal relationship extraction and classified different ways to express causal relationships in English text:

1. Causal links are typical words or phrases that link cause and effect, e.g. 'hence', 'therefore', 'because of', 'on account of', etc. The different classifications of causal links were based on Altenberg [3].

2. Causal verbs are verbs that mean to cause something, be caused by something, prevent something from happening, or affect something, e.g. 'lead to', 'trigger', 'enable' (based on Thomson [48]).

3. Resultative constructions refer to sentences where the object of a verb is followed by a phrase that describes the state of the object as a result of the action implied by the verb, e.g. 'I painted the car yellow.' These constructions are based on Simpson [43].

4. Conditionals often indicate a causal relationship where the antecedent (the if part) causes the consequent (the then part) (based on [11]).

5. Causal adverbs and adjectives often have a causal element in their meaning, e.g. 'Brutus fatally wounded Caesar' (based on [10]).

Girju and Moldovan [15] presented an approach for the automatic detection of causal patterns and semiautomatic validation of ambiguous lexico-syntactic patterns referring to causation. One of the results is a list of causal verbs containing words like 'generate', 'associate', 'originate', etc. ordered by ambiguity and frequency which is often used by other researchers. This approach was novel insofar as linguistic patterns were extracted semi-automatically and no longer by hand as it was done in previous research. The accuracy on a corpus of news articles was 0.65 .

Sorgente et. al [44] worked on techniques that incorporated different variations of machine learning approaches. They designed a hybrid system relying on rules and a Bayesian classifier. Precision and recall were between 0.6 and 0.7 .

Ackermann [1] compared among other aspects different machine learning techniques on a set of New York Times news articles. F-measures were 0.74 for Naive Bayes, 0.78 for dictionary classifiers, 0.58 for KNN, 0.67 for SVM, 0.70 for decision trees and 0.71 for neural networks.

Yang and Mao [50] as well as Pakray and Gelbukh [40] developed additional machine learning systems achieving F-measures between 0.65 and 0.85 for the detection of causal sentences.

In the related work, rule-based papers used pattern matching, linguistic clues, cue phrases, and causal verbs as methods for causal information extraction [6]. The main machine learning methods applied were decision trees, SVM, and Naïve Bayes [6].

Most corpora in the related work were derived from relatively broad sources like newspapers or Wikipedia. Most of the research regarding causal relationship extraction focuses on domain-independent approaches for general corpora [6]. Also, the prior research mainly checks for causality but rarely addresses the extraction of causal variables beyond cause and effect, e.g. moderator and mediator variables as well as causal directions between constructs. 


\section{CauseMiner}

CauseMiner is a software that analyzes text in order to identify and extract sentences containing causal relationships. It detects, analyzes, and extracts hypotheses and propositions from research papers. CauseMiner is developed and tested on papers that belong to the Senior Scholars' Basket of Journals [7]. The decision for these papers is based on the relevance for the Information Systems research community and the domain expertise of the authors.

CauseMiner extracts a corpus of TXT-files (after conversion from PDF to text) and saves results into a CSV-file. It applies a rule-based approach and is written in Python. Rule-based approaches are still quite effective for some complex information extraction tasks without a lot of annotated data [6, 20, 34] and could also be combined with machine learning approaches within hybrid systems $[6,20]$.

\subsection{Pre-Processing}

Figure 2 provides an abstract overview of the preprocessing steps. The analyzed papers are available as PDF and converted into text format with the open source software pdftotext [16]. This conversion of PDF files into text causes significant problems regarding data quality. After conversion, text includes page breaks or header/footer information that would impede further processing. An analysis of conversion errors revealed variations of possible data problems for each journal. Figure 3 provides two examples of conversion errors that interrupt the text-flow.

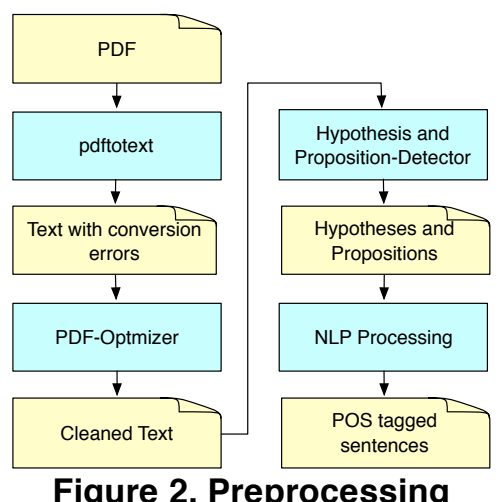

Because the name of a journal isn't necessarily part of the filename nor otherwise indicated, CauseMiner detects the name of a journal per regular expressions and executes specific clean up procedures, e.g. removal of header/footer information, concatenation of page breaks, removal of references, figures, etc. This is achieved by a set of rules for each journal, e.g. if the actual line is empty, check if seven lines later only 1-4 numbers occur. If yes, remove the lines in-between. These rules can be easily adjusted for other journals that are not part of the AIS basket of eight.

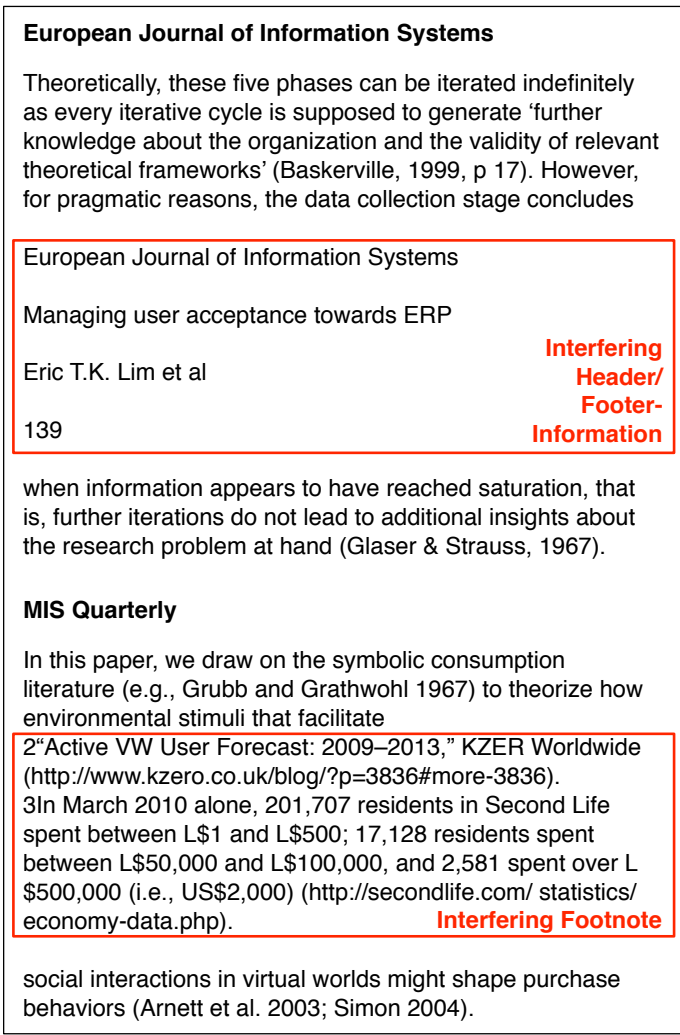

Figure 3. PDF-conversion errors [4, 35]

The analysis of embedded hypotheses in text data reveals many variations. Each journal has a specific style of expressing hypotheses and propositions that can additionally vary depending on the author's preferences. Explicitly expressed hypotheses and propositions are almost always preceded by an indicator, e.g. "H1:" or "P2a:". However, variations are manifold: "Hypothesis 3 (H3).", "P3A.", "Proposition 3.1:", "H1a-e:", "P1", etc. CauseMiner detects hypotheses and propositions after the text cleaning procedure via regular expression. Because of the many possible variations of different indicators, the design of a robust regular expression for this task is difficult.

Therefore, the implemented regex is kind of overtolerant in order to catch all possible variations. The implemented regex looks like this:

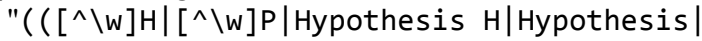
Proposition P|Proposition) $\backslash \mathrm{s}$ ? $([0-9]+[\backslash$.$] ? [0-$ $9] *[a A-z Z]$ ? $)$ ? $(\backslash(. * ? \backslash))$ ? [.: ]*[\s]+(.*?[a$\mathrm{z}]\{0\},[]$.$) ? \backslash \mathrm{W}^{*}$ ? $)^{\prime \prime}$

However, catching all occurrences of different types of hypotheses statements in a paper is 
problematic especially when the regex catches not only the initial hypotheses but also snippets from the result section, e.g. "H1a: $\mathrm{p}<0.01$ ". To address this overtolerance, additional logic is applied where the prototype tests, if e.g. the extracted contents are numbers or letters. Only valid hypotheses and propositions are processed further.

Parsing detected hypotheses and propositions allows for the application of more sophisticated rules, e.g. filtering for words and word types. CauseMiner utilizes SpaCy [21], a Python library for natural language processing that is fast and has an accuracy within $1 \%$ of the best available NLP libraries $[9,21$, 22]. Each hypothesis and proposition is parsed into separate word objects and each of these objects is enriched with index-, string-, lemma-, and POSinformation. Figure 4 illustrates the parsing of a hypothesis.

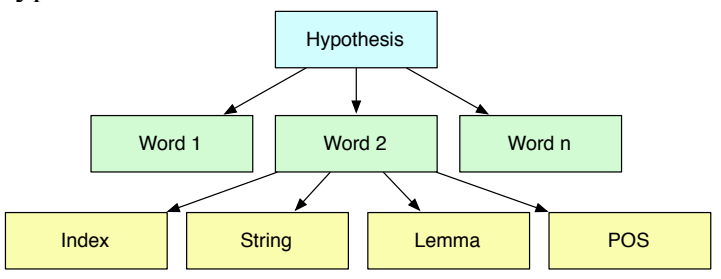

Figure 4. Hypothesis parsing

\subsection{Information Extraction Process}

An analysis of extracted hypotheses and propositions showed that conditions typically appear at the beginning or end of a sentence. Therefore, they are extracted first. The extracted part is cut off from the sentence and only remaining words are processed further. Moderator and mediator variables are extracted next and again, only the remaining words are analyzed further. Lastly, cause and effect are extracted. This extraction order is the result of experimentations with different rule variations and architecture designs. This is in line with design as a search process according to Hevner [19]. Starting with the extraction of causal patterns resulted for example in complications with extracting conditions later on, because each extracted variable had to be checked separately. Cutting already extracted parts off the sentence during the process prevented redundancy in the code and proved to be more robust. Figure 5 illustrates the extraction process.

For extracting variables as separated constructs, several extraction-patterns were developed. The goal was to extract hypotheses and propositions into the meta-model of Mueller [38] consisting of cause, effect, sign, mediator, moderator, interaction sign, and condition. Therefore, the authors manually extracted 274 papers of the basket of eight and copied all detected 963 hypotheses and propositions into a spreadsheet (see Section 5.1 for more details on the corpora). For every hypothesis and proposition, all variables (cause, effect, etc.) were stored separately into designated columns in the same spreadsheet.

This manual extraction revealed different types of causal statements that were clustered based on the results of former research in this area. The resulting categories or patterns were the basis for the development of extraction procedures within the prototype.

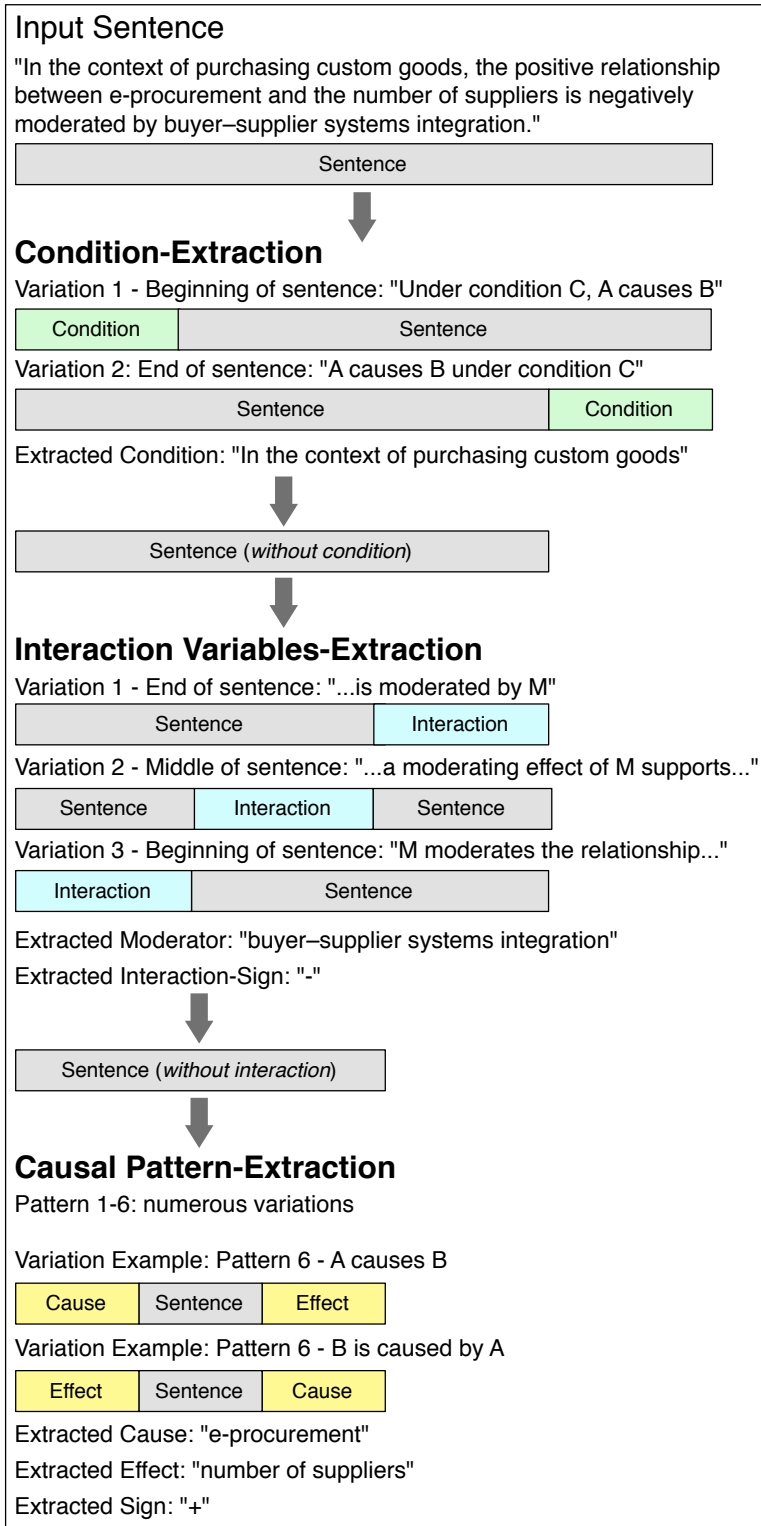

Figure 5. Hypothesis extraction

Table 1 provides an overview of the extraction patterns. The example pattern column contains an abstract example of the underlying rules: brackets hold examples of keywords, $C_{i}$ stands for construct, rel for 
Table 1. Causal extraction patterns

\begin{tabular}{|c|c|c|c|}
\hline Pattern Name & Example Pattern & Example Sentence & Based on \\
\hline $\begin{array}{l}\text { Causal } \\
\text { Relationships }\end{array}$ & $\begin{array}{l}\text { [relationship, } \\
\text { interaction, linkage, } \\
\text { etc. between] } \mathrm{C}_{1} \text { and } \mathrm{C}_{2}\end{array}$ & $\begin{array}{l}\text { "H2: MTA positively moderates the relationship between perceived } \\
\text { usefulness (adoption) and intention to use." [46] }\end{array}$ & \\
\hline $\begin{array}{l}\text { Causal } \\
\text { Keywords }\end{array}$ & $\begin{array}{l}\mathrm{C}_{1} \text { [because, as, since, } \\
\text { etc.] } \mathrm{C}_{2}\end{array}$ & $\begin{array}{l}\text { "H2a: When using audio, unilateral concessions will increase due to the } \\
\text { communication of fact-related questions." }[23]\end{array}$ & {$[3,25]$} \\
\hline $\begin{array}{l}\text { Causal } \\
\text { Comparisons }\end{array}$ & $\begin{array}{l}\mathrm{C}_{1} \text { rel } \mathrm{C}_{2} \text { [greater than] } \\
\mathrm{C}_{1} \text { rel } \mathrm{C}_{3}\end{array}$ & $\begin{array}{l}\text { "Hypothesis } 5 \mathrm{~b} \text { (H5b). Firms that use advanced BA will not generate } \\
\text { higher positive abnormal returns than firms that use basic BA." [47] }\end{array}$ & {$[42]$} \\
\hline $\begin{array}{l}\text { Causal } \\
\text { Correlations }\end{array}$ & $\begin{array}{l}\text { [the more, higher, } \\
\text { faster] } \mathrm{C}_{1},[\text { the lower, } \\
\text { fewer, less }] \mathrm{C}_{2}\end{array}$ & $\begin{array}{l}\text { "Hypothesis 3: The higher an employee's resistance to change, the } \\
\text { higher her user resistance behaviour." [32] }\end{array}$ & {$[42]$} \\
\hline Causal Verbs & $\begin{array}{l}\mathrm{C}_{1} \text { [causes, affects, } \\
\text { increases] } \mathrm{C}_{2}\end{array}$ & $\begin{array}{l}\text { "Hypothesis 2: Cohesive argument structuring relates positively to } \\
\text { deception severity in CMC." [36] }\end{array}$ & {$[15,48]$} \\
\hline Conditions & $\begin{array}{l}\text { If Cond., } \mathrm{C}_{1} \text { [causes] } \\
\mathrm{C}_{2} ; \text { If Cond., rel } \\
\text { between } \mathrm{C}_{1} \text { and } \mathrm{C}_{2}\end{array}$ & $\begin{array}{l}\text { "Hypothesis } 2(\mathrm{H} 2 \mathrm{~A}) \text {. In contexts in which initial technology trust } \\
\text { expectations are more mature, positive trust disconfirmation will } \\
\text { negatively influence trusting intention." [28] }\end{array}$ & \\
\hline $\begin{array}{l}\text { Moderator, } \\
\text { Mediator }\end{array}$ & $\begin{array}{l}\mathrm{M} \text { [moderates, } \\
\text { mediates] rel between } \\
\mathrm{C}_{1} \text { and } \mathrm{C}_{2}\end{array}$ & $\begin{array}{l}\text { "H2a. The relationship between the level of e-government development } \\
\text { in a country and the extent of corruption in its national business service } \\
\text { systems is mediated by the corruption in its national political } \\
\text { institutions." [45] }\end{array}$ & \\
\hline
\end{tabular}

relation. Each pattern includes numerous variations, e.g. distinction between active and passive sentences, sign detection (positive, negative, not mentioned, negated) or detection of patterns in different parts of a sentence (beginning, middle, end).

The example patterns shown in Table 1 are just small excerpts that illustrate the general rule-logic. Each pattern includes many variations: the pattern "Causal Verbs" includes, for example, grammatical variations such as $" \mathrm{C}_{1}$ causes/caused/will cause/is going to cause/etc. $\mathrm{C}_{2}$ " as well as " $\mathrm{C}_{2}$ is caused by/has been caused by/was caused by/etc. $\mathrm{C}_{1}$ ". It also includes different keywords such as "impacts", "influences", "increases", "stimulates", etc.

After extraction, CauseMiner removes stop words from constructs and saves results in table-form in a CSV-file. The CSV-files (or alternatively a different table-format or a database) are used to run automated tests that compare annotated CSV-files with the results of the prototype. It also allows further processing of single variables in later development stages that might be covered in a future version of CauseMiner (e.g. matching of synonyms).

The fieldnames in this file are "HypothesisNumber", "Hypothesis/Proposition", "PatternNumber", "Cause", "Effect", "Sign", "Condition", "Moderator", "Mediator", "Interaction-Sign", "Journal", "Author", "Year", "Title", and some binary control variables. For example, the hypothesis "MTA positively moderates the relationship between perceived usefulness (adoption) and intention to use." [46] would be saved as:

- Hypothesis-Number: '3a'

- Hypothesis: 'MTA positively moderates the relationship between perceived usefulness (adoption) and intention to use.'

- Pattern: '1'

- Cause: 'perceived usefulness (adoption)'

- Effect: 'intention to use'

- Sign: '?'

- Condition_Flag: 'False'

- Condition: '

- Interaction_Flag: 'True'

- Moderator: 'MTA'

- Mediator: ' '

- Interaction Sign: '+'

- Journal: 'Journal of the Association for Information Systems'

- Author: 'Sun et. al'

- Year: '2016'

- Title: 'Choosing a Fit Technology: Understanding Mindfulness in Technology Adoption and Continuance'

While iterating over a corpus of papers, CauseMiner adds one row for each detected hypothesis/proposition into a CSV-file that can be further analyzed.

\section{Evaluation}

\subsection{Corpus Creation}

In order to test the prototype for the Information Systems domain, the authors manually analyzed three corpora and created several goldmaster files. The annotation process consisted of manually analyzing PDF-files and extracting explicitly stated hypotheses and propositions into an Excel-sheet - a goldmaster file. For each hypothesis, the following variables were 
placed into separate columns: hypothesis, hypothesisnumber, cause, effect, causal sign, moderator, mediator, interaction sign, condition, journal, author, title.

This process was conducted by two annotators (the authors): (1) creation of an annotation protocol, (2) collection of papers that belong to the basket of eight: For each year (2007-2015), the amount of papers should be equally distributed, (3) manual checking of papers for the existence of hypotheses/propositions and the documentation of findings in a CSV file (this step was done once by each annotator): If a hypothesis/proposition was found, all relevant variables were manually extracted in the provided CSV fields, and (4) discussion of deviations regarding the annotators judgments: if manual extraction-results deviated from each other, the annotators agreed on a final desired extraction result.

The first corpus consisted of 124 papers containing 474 hypotheses and propositions. It led to the development and implementation of an initial rule-set. The second corpus consisted of 150 papers containing 489 hypotheses and propositions. This second corpus helped to refine and optimize the initial rules. All papers belonged to journals of the AIS basket of eight [7] and were published from 2008 till 2015. The third corpus was designed to evaluate the improved rulebased approach and was created after the rule-based system was finalized. This corpus contains all hypotheses of all 270 papers published in 2016 in the AIS basket of eight containing 723 hypotheses and propositions.

\subsection{Evaluation}

The extraction method was tested against the manually created goldmaster file. Table 2 provides an overview of the test corpus from 2016 for the evaluation step. Table 3 shows the distribution of the causal extraction patterns in the test corpus.

Table 2. Goldmaster corpus overview

\begin{tabular}{|lll|}
\hline Detection Results & Total & Rel. \\
\hline Papers in corpus & 270 & 1.0 \\
Papers without causal claims & 154 & 0.57 \\
Papers containing causal claims & 116 & 0.43 \\
\hline
\end{tabular}

For each variable, true positives, false positives, true negatives, false negatives, precision, recall, Fmeasure, and accuracy were computed. Table 4 contains the definitions for all measures. Measures for variables (cause, effect, etc.) were only computed for those hypotheses that were detected.

When CauseMiner did not detect a hypothesis that should have been detected, only false positives for the hypothesis-variable were affected, but not for other variables. Only exact matches are considered true positives. Because one missing word in a construct might drastically change the meaning of the construct, partial matches are considered as false.

Table 3. Causal Extraction Pattern Distribution

\begin{tabular}{|lll|}
\hline Detection Results & Total & Rel. \\
\hline Extraction patterns were applied & 564 & 0.82 \\
Extraction patterns couldn't be applied & 127 & 0.18 \\
\hline \hline Extracted Pattern & Total & Rel. \\
\hline Causal Relationships & 104 & 0.18 \\
Causal Keywords & 25 & 0.04 \\
Causal Comparisons & 54 & 0.10 \\
Causal Correlations & 61 & 0.11 \\
Causal Verbs & 287 & 0.51 \\
Other (e.g. condition + effect, no cause) & 33 & 0.06 \\
\hline \hline Additional Pattern & Total & Rel. \\
\hline Conditions & 65 & 0.09 \\
Moderator & 27 & 0.04 \\
Mediator & 9 & 0.01 \\
\hline
\end{tabular}

Table 4. Measures for evaluation

\begin{tabular}{|c|c|}
\hline Measure & Description \\
\hline $\begin{array}{l}\text { True } \\
\text { positive } \\
(\mathrm{TP})\end{array}$ & $\begin{array}{l}\text { Variable in goldmaster-file equals variable in } \\
\text { computed output and is not empty ('found } \\
\text { something and it is correct') }\end{array}$ \\
\hline $\begin{array}{l}\text { False } \\
\text { positive } \\
(\mathrm{FP})\end{array}$ & $\begin{array}{l}\text { Variable in goldmaster-file doesn't equal } \\
\text { variable in computed output ('found } \\
\text { something but it was the wrong thing') }\end{array}$ \\
\hline $\begin{array}{l}\text { True } \\
\text { negative } \\
(\mathrm{TN})\end{array}$ & $\begin{array}{l}\text { Both variables in goldmaster-file and in } \\
\text { computed output are empty ('found nothing } \\
\text { and it is correct') }\end{array}$ \\
\hline $\begin{array}{l}\text { False } \\
\text { negative } \\
(\mathrm{FN})\end{array}$ & $\begin{array}{l}\text { Variable in computed output is empty, but } \\
\text { variable in goldmaster-file is not ('found } \\
\text { nothing although something should have } \\
\text { been found') }\end{array}$ \\
\hline Precision & $\frac{\mathrm{TP}}{\mathrm{TP}+\mathrm{FP}}$ \\
\hline Recall & $\frac{\mathrm{TP}}{\mathrm{TP}+\mathrm{FN}}$ \\
\hline $\begin{array}{l}\text { F-Measure } \\
\text { (F1-Score) }\end{array}$ & $2 \cdot \frac{\text { Precision } \cdot \text { Recall }}{\text { Precision }+ \text { Recall }}$ \\
\hline Accuracy & $\frac{\mathrm{TP}+\mathrm{TN}}{\mathrm{TP}+\mathrm{TN}+\mathrm{FP}+\mathrm{FN}}$ \\
\hline
\end{tabular}

Table 5 shows the comparison of CauseMiner's output against the annotated goldmaster file. In the hypothesis detection task, CauseMiner achieved 0.88 precision, 0.88 recall, $0.88 \mathrm{~F}$-measure, and 0.82 accuracy. Compared to the hypotheses detection of $\mathrm{Li}$ $\&$ Larsen [33] (precision 0.85, recall: 0.85, F-Measure: 0.85 ) these are slightly better results.

The results of the causal relationship extraction of CauseMiner are only to a limited amount comparable 
to the related work, because not only cause and effect, but also sign, moderator, mediator, condition, and interaction sign were extracted. Another limitation regarding the comparability of the results is that the related work mainly uses different corpora often from news and medical abstracts. Also, not every paper published all relevant metrics like precision, recall, Fmeasure, and accuracy but just a subset or only one, like accuracy or precision.

Table 5. Evaluation results

\begin{tabular}{|lllll|}
\hline Variable & TP & FP & FN & TN \\
\hline Hypothesis & 616 & 82 & 85 & 148 \\
Cause & 401 & 108 & 109 & 26 \\
Effect & 408 & 122 & 95 & 19 \\
Sign & 513 & 17 & 95 & 19 \\
Condition & 60 & 5 & 50 & 529 \\
Moderator & 27 & 0 & 17 & 600 \\
Mediator & 9 & 0 & 12 & 623 \\
Int.-Sign & 36 & 0 & 29 & 579 \\
\hline \hline Variable & Prec. & Recall & F1 & Acc. \\
\hline Hypothesis & 0.88 & 0.88 & 0.88 & 0.82 \\
Cause & 0.79 & 0.79 & 0.79 & 0.66 \\
Effect & 0.77 & 0.81 & 0.79 & 0.66 \\
Sign & 0.97 & 0.84 & 0.90 & 0.83 \\
Condition & 0.92 & 0.55 & 0.69 & 0.91 \\
Moderator & 1.0 & 0.61 & 0.76 & 0.97 \\
Mediator & 1.0 & 0.43 & 0.60 & 0.98 \\
Int.-Sign & 1.0 & 0.55 & 0.71 & 0.95 \\
\hline
\end{tabular}

\subsection{Error Analysis and Limitations}

An error analysis showed different categories and sources whereas none of them are regarded as major issues. Nearly all of them could be resolved by optimizing different parts of the prototype. Table 6 provides an overview of some typical errors.

Other problems refer, for example, to multiple variables. The following hypothesis contains actually four hypotheses in one sentence: "H1: For information search via mobile Web browsing, the tree-view presentation adaptation will lead to (a) reduction in search time, (b) increase in search accuracy, (c) increase in perceived ease of use, and (d) increase in perceived usefulness." [2]. This hypothesis contains multiple causal statements and is therefore difficult to extract.

Hypotheses and propositions containing mathematical expressions are problematic as well. Due to the pdf to text conversion, mathematical symbols are converted to Unicode and this results in hieroglyphic statements that can't be processed any further, e.g. "The bettor i's optimal betting strategy is ri* $=\mathrm{E}[\mathrm{X} \mid \mathrm{si}$ ]$=\tau 0 \mathrm{~s} 0+\tau \mathrm{i}$ si $\tau 0+\tau \mathrm{i} \mathrm{Bi}=\tau \mathrm{i} 2 \tau 02(\tau 0+\tau \mathrm{i}) 2$ with a positive expected payoff $\tau \mathrm{i} 2(\tau 0+\tau \mathrm{i}) 2$. ." [14].
Some semantic challenges must also be addressed, for example hypotheses that contain statements of comparison: "When using a KBS with explanation facilities, experts will choose more feedback explanations than novices." [5]. Currently, CauseMiner extracts a condition ('When using a KBS with explanation facilities'), a cause ('experts'), an effect ('feedback explanations') and a sign ('+'). But the comparison referring to novices is not extracted.

Table 6. Error examples

\begin{tabular}{|l|l|l|}
\hline Category & Expected & Retrieved \\
\hline $\begin{array}{l}\text { Sentence } \\
\text { Boundary } \\
\text { Detection }\end{array}$ & $\begin{array}{l}\text { "Perceived effectiveness of } \\
\text { transaction intention is } \\
\text { stronger for the U.S.A. } \\
\text { consumers than for Korean } \\
\text { consumers." }\end{array}$ & $\begin{array}{l}\text { "Perceived } \\
\text { effectiveness of } \\
\text { WASS on e- } \\
\text { commerce } \\
\text { transaction } \\
\text { intention is } \\
\text { stronger for the } \\
\text { U." }\end{array}$ \\
\hline $\begin{array}{l}\text { Encoding } \\
\text { Errors } \\
\text { (wrong } \\
\text { apostro- } \\
\text { phe sign) }\end{array}$ & $\begin{array}{l}\text { "The higher an IT worker's } \\
\text { agreeableness, the higher } \\
\text { her organizational }\end{array}$ & $\begin{array}{l}\text { "The higher an } \\
\text { IT worker's } \\
\text { agreeableness, } \\
\text { the higher her } \\
\text { organizational } \\
\text { commitment." }\end{array}$ \\
\hline $\begin{array}{l}\text { Not } \\
\text { ignoring } \\
\text { parenthe- } \\
\text { sis }\end{array}$ & $\begin{array}{l}\text { "For both static and } \\
\text { polymorphic warnings, } \\
\text { attention will decrease in } \\
\text { terms of mouse cursor } \\
\text { movements (greater area } \\
\text { under the curve, slower } \\
\text { average speed, and slower } \\
\text { initial acceleration) across } \\
\text { repeated exposures." }\end{array}$ & $\begin{array}{l}\text { Extracted } \\
\text { cause: "area } \\
\text { under the } \\
\text { curve" }\end{array}$ \\
\hline $\begin{array}{l}\text { Cause: } \\
\text { "Paradox-framed } \\
\text { trajectories" }\end{array}$ & $\begin{array}{l}\text { Extracted } \\
\text { ting } \\
\text { errors }\end{array}$ & $\begin{array}{l}\text { "Paradox- } \\
\text { framed } \\
\text { trajectories are } \\
\text { strongly" }\end{array}$ \\
\hline
\end{tabular}

\section{Conclusion and Outlook}

This paper presents a novel method and a working prototype that automatically extracts not only causes and effects but also signs, moderators, mediators, conditions, and interaction signs from hypotheses and propositions published in scientific papers. Additionally, a review of related literature was conducted that shows that most of the related work uses either news articles or medical papers as a corpus. This paper specifically addresses Information Systems papers of the AIS basket of eight for developing and testing its approach. For the task of theory ontology learning and theory integration this corpus is regarded more relevant than, for example, news articles. 
Due to the limited sample size and the nature of the corpus it is hard to make claims regarding the generalizability of the achieved results. However, as the prototype was developed and fine-tuned by developing two corpora consisting of 274 papers containing 963 hypotheses and propositions in total, as well as being tested over a corpus of 270 papers with 723 hypotheses, the results are promising - especially because the prototype proved to be robust enough to catch the majority of expressions in a domain known for rather complex phrasing.

Construct matching was not a part of this paper, but shall be implemented in the future. Larsen and Bong [30] developed a hybrid method for construct detection based on latent semantic analysis (LSA) and knowledge-based methods. In future work, a similar construct matching approach could be used to further analyze the output of CauseMiner.

CauseMiner is sensitive to errors during the preprocessing and extraction steps (e.g. errors in the PDF parsing or part-of-speech tagging) which can lead to wrongly formatted input for additional analytical techniques. Further optimization should make CauseMiner more robust for these kinds of errors.

Asghar [6:8] found no paper that used Deep Neural Networks for causal relationship extraction but suggests it might be effective because of the feature abstraction capabilities. This might be an interesting future approach for CauseMiner.

Furthermore, a hybrid combination of CauseMiner's rule-based approach with machine learning methods might be a promising future possibility.

In the future, CauseMiner will be used to analyze the Information Systems field from the last 20 years including construct matching [30]. CauseMiner could then be used to describe papers based on a theory ontology. This would enable research to analyze and integrate many theories with, for example, theoryevolution graphs [38] or theory-data maps [39].

\section{References}

[1] Ackerman, E.J.M. Extracting Causal Relations between News Topics from Distributed Sources. Dissertation, Technische Universität Dresden (2013).

[2] Adipat, B., Zhang, D., and Zhou, L. The effects of treeview based presentation adaptation on mobile web browsing. MIS Quarterly 35, 1 (2011), 99-121.

[3] Altenberg, B. Causal Linking in Spoken and Written English. Studia Linguistica 38, 1 (1984), 20-69.

[4] Animesh, A., Pinsonneault, A., Yang, S.-B., and Oh, W. An odyssey into virtual worlds: exploring the impacts of technological and spatial environments on intention to purchase virtual products. MIS Quarterly 35, 3 (2011), 789-
810.

[5] Arnold, V., Clark, N., Collier, P.A., Leech, S.A., and Sutton, S.G. The differential use and effect of knowledgebased system explanations in novice and expert judgment decisions. MIS Quarterly 30, 1 (2006), 79-97.

[6] Asghar, N. Automatic Extraction of Causal Relations from Natural Language Texts: A Comprehensive Survey. arXiv preprint arXiv:1605.07895, (2016).

[7] Association for Information Systems. Website - Senior Scholars' Basket of Journals. 2017.

https://aisnet.org/SeniorScholarBasket.

[8] Baron, R.M. and Kenny, D.A. The moderator-mediator variable distinction in social psychological research:

Conceptual, strategic, and statistical considerations. Journal of personality and social psychology 51, 6 (1986), 1173.

[9] Choi, J.D., Tetreault, J.R., and Stent, A. It Depends: Dependency Parser Comparison Using A Web-based Evaluation Tool. $A C L$ (1), (2015), 387-396.

[10] Cresswell, M.J. Adverbs of Causation. In Adverbial Modification. Springer Netherlands, Dordrecht, 1981, 173192.

[11] Cummins, D.D., Lubart, T., Alksnis, O., and Rist, R. Conditional reasoning and causation. Memory \& cognition 19, 3 (1991), 274-282.

[12] Davis, R., Campbell, R., Hildon, Z., Hobbs, L., and Michie, $\mathrm{S}$. Theories of behaviour and behaviour change across the social and behavioural sciences: a scoping review. Health Psychology Review 9, 3 (2015), 323-344.

[13] Dedrick, J., Xu, S.X., and Zhu, K.X. How Does Information Technology Shape Supply-Chain Structure? Evidence on the Number of Suppliers. Journal of Management Information Systems 25, 2 (2008), 41-72.

[14] Fang, F., Guo, Z., and Whinston, A.B. Collective Outsourcing to Market (COM): A market-based framework for information supply chain outsourcing. Journal of the Association for Information Systems 9, 3/4 (2008), 98-118.

[15] Girju, R. and Moldovan, D. Text mining for causal relations. Proceedings of the FLAIRS Conference, (2002), 360-364.

[16] Glyph \& Cog. Website - Xpdf Project. 2017. http://www.foolabs.com/xpdf/about.html.

[17] Gregor, S. The nature of theory in information systems. MIS quarterly 30, 3 (2006), 611-642.

[18] Gregor, S. and Hovorka, D. Causality: The elephant in the room in information systems epistemology. Proceeding of the European Conference on Information Systems (ECIS), (2011).

[19] Hevner, A.R., March, S.T., Park, J., and Ram, S. Design science in information systems research. MIS Quarterly 28, 1 (2004), 75-105.

[20] Hogenboom, F., Frasincar, F., Kaymak, U., de Jong, F., and Caron, E. A Survey of event extraction methods from text for decision support systems. Decision Support Systems 85 , (2016), 12-22.

[21] Honnibal, H. Website - spaCy: Industrial-Strength Natural Language Processing. 2017. https://spacy.io. 
[22] Honnibal, M. and Johnson, M. An Improved Nonmonotonic Transition System for Dependency Parsing. EMNLP, (2015), 1373-1378.

[23] Johnson, N.A., Cooper, R.B., and Holowczak, R.D. The impact of media on how positive, negative, and neutral communicated affect influence unilateral concessions during negotiations. European Journal of Information Systems 25, 5 (2016), 391-410.

[24] Khoo, C. Automatic Identification of Causal Relations in Text and their use for Improving Precision in Information Retrieval - PhD Thesis - Syracuse University. 1995.

[25] Khoo, C., Chan, S., and Niu, Y. The many facets of the cause-effect relation. In The Semantics of Relationships. Springer, 2002, 51-70.

[26] Khoo, C.S., Chan, S., and Niu, Y. Extracting causal knowledge from a medical database using graphical patterns. Proceedings of the 38th Annual Meeting on Association for Computational Linguistics, (2000), 336-343.

[27] Khoo, C.S., Kornfilt, J., Oddy, R.N., and Myaeng, S.H. Automatic extraction of cause-effect information from newspaper text without knowledge-based inferencing. Literary and Linguistic Computing 13, 4 (1998), 177-186.

[28] Lankton, N.K., McKnight, D.H., Wright, R.T., and Thatcher, J.B. Using Expectation Disconfirmation Theory and Polynomial Modeling to Understand Trust in Technology. Information Systems Research 27, 1 (2016), 197-213.

[29] Larsen, K., Lee, J., Li, J., and Bong, C. A Transdisciplinary Approach to Construct Search and Integration. AMCIS 2010 Proceedings, (2010).

[30] Larsen, K.R. and Bong, C.H. A tool for addressing construct identity in literature reviews and meta-analyses. MIS Quarterly 40, 3 (2016), 1-23.

[31] Larsen, K.R., Hovorka, D., West, J., et al. Theory Identity: A Machine-Learning Approach. 47th Hawaii International Conference on System Sciences (HICSS), IEEE (2014), 4639-4648.

[32] Laumer, S., Maier, C., Eckhardt, A., and Weitzel, T. User personality and resistance to mandatory information systems in organizations: a theoretical model and empirical test of dispositional resistance to change. Journal of Information Technology 31, 1 (2016), 67-82.

[33] Li, J. and Larsen, K. Establishing Nomological Networks for Behavioral Science: a Natural Language Processing Based Approach. ICIS 2011 Proceedings, (2011). [34] Lim, C.-G. A survey of temporal information extraction and language independent features. International Conference on Big Data and Smart Computing (BigComp), (2016), 447449.

[35] Lim, E.T.K., Pan, S.L., and Tan, C.W. Managing user acceptance towards enterprise resource planning (ERP) systems - understanding the dissonance between user expectations and managerial policies. European Journal of Information Systems 14, 2 (2005), 135-149.

[36] Ludwig, S., van Laer, T., de Ruyter, K., and Friedman,
M. Untangling a Web of Lies: Exploring Automated Detection of Deception in Computer-Mediated Communication. Journal of Management Information Systems 33, 2 (2016), 511-541.

[37] Michels, C. and Schmoch, U. The growth of science and database coverage. Scientometrics 93, 3 (2012), 831-846.

[38] Mueller, R.M. A Meta-Model for Inferring Inter-Theory Relationships of Causal Theories. Proceedings of the 48th Hawaii International Conference on System Sciences (HICSS). IEEE Computer Society Press, (2015), 4908-4917.

[39] Mueller, R.M. Theory-Data Maps: A Meta-Model and Methods for Inferring and Visualizing Relationships between Causal Theories and Empirical Evidences. Proceedings of the 49th Hawaii International Conference on System Sciences (HICSS). IEEE Computer Society Press, (2016), 5288-5297.

[40] Pakray, P. and Gelbukh, A. An Open-Domain CauseEffect Relation Detection from Paired Nominals. In A. Gelbukh, F.C. Espinoza and S.N. Galicia-Haro, eds., NatureInspired Computation and Machine Learning. Springer International Publishing, 2014, 263-271.

[41] Pearl, J. The causal foundations of structural equation modeling. In Handbook of Structural Equation Modeling. Guilford Press, New York, 2012.

[42] Sevastopoulos, J. Website - Grammar Quizzes, English Grammar Reference: Resource, Practices \& Exercises. 2017. http://www.grammar-quizzes.com.

[43] Simpson, J. Resultatives. In L. Levin, M. Rappaport and A. Zaenen, eds., Papers in Lexical-functional Grammar. Bloomington: Indiana University Linguistics Club, 1983, 143-157.

[44] Sorgente, A., Vettigli, G., and Mele, F. Automatic Extraction of Cause-Effect Relations in Natural Language Text. Proceedings of the 7th International Workshop on Information Filtering and Retrieval-DART@AI*IA 2013, (2013), 37-48.

[45] Srivastava, S.C., Teo, T.S., and Devaraj, S. You Can't Bribe a Computer: Dealing with the Societal Challenge of Corruption Through ICT. MIS Quarterly 40, 2 (2016), 511526.

[46] Sun, H., Fang, Y., and others. Choosing a Fit Technology: Understanding Mindfulness in Technology Adoption and Continuance. Journal of the Association for Information Systems 17, 6 (2016), 377-412.

[47] Teo, T.S.H., Nishant, R., and Koh, P.B.L. Do shareholders favor business analytics announcements? The Journal of Strategic Information Systems 25, 4 (2016), 259276.

[48] Thomson, J.J. Verbs of action. Synthese 72, 1 (1987), 103-122.

[49] Weber, R. Evaluating and Developing Theories in the Information Systems Discipline. Journal of the Association for Information Systems 13, 1 (2012), 1-30.

[50] Yang, X. and Mao, K. Multi level causal relation identification using extended features. Expert Systems with Applications 41, 16 (2014), 7171-7181. 